\title{
Surgical removal of a migrating intraspinal bullet: illustrative case
}

\author{
*Daniel de los Cobos, BA, ${ }^{1}$ Alexa Powers, BA, ${ }^{1}$ Jonathan P. Behrens, MD, ${ }^{1}$ Tobias A. Mattei, MD, ${ }^{2}$ and Pooria Salari, MD ${ }^{1}$ \\ Departments of ${ }^{1}$ Orthopaedic Surgery and ${ }^{2}$ Neurosurgery, Saint Louis University School of Medicine, St. Louis, Missouri
}

BACKGROUND Management of gunshot wounds to the spine with subsequent spinal cord injury is a controversial topic among spine surgeons. Possible complications of retained intradural bullets include delayed neurological deficits, spinal instability, and lead toxicity. The authors' purpose is to review the potential complications of retained intraspinal bullets and the surgical indications for intraspinal bullet removal.

OBSERVATIONS The authors describe a case of a patient who developed cauda equina symptoms following a gunshot wound to the lumbar spine with a migrating retained intraspinal bullet. Because of neurological changes, the patient underwent surgical removal of the bullet. At the postoperative clinic visit 2 weeks following bullet removal, the patient reported resolution of her symptoms.

LESSONS Gunshot wounds to the spine are challenging cases. The decision to proceed with surgical management in the event of retained bullet fragments is multifactorial and relies heavily on the patient's neurological status. A current review of the literature suggests that, in cases of cauda equina injuries and the development of neurological deficits in patients with retained intraspinal fragments, there is benefit from surgical decompression and bullet removal. Careful preoperative planning is required, and consideration of spinal alignment with positional changes is crucial.

https://thejns.org/doi/abs/10.3171/CASE21132

KEYWORDS gunshot wounds; spinal cord injury; cauda equina; migrating bullet

Civilian gunshot wounds (GSWs) to the spine have become an increasingly common occurrence at trauma centers in the United States. GSWs are the third leading cause of all spinal cord injuries (15.2\%) after automobile accidents (38.6\%) and falls (22.9\%). ${ }^{1}$ These injuries occur predominantly among the younger male population and are commonly related to criminal or violent activities with a predilection for urban areas. Damage to the spinal cord from GSWs often results in complete injuries caused by cord transection or vascular disruption.

The thoracic spine is the most commonly involved level (51.8\%), followed by the thoracolumbar spine $(28.9 \%)$ and the cervical spine $(19.3 \%)^{2}{ }^{2}$ The location of the lesion determines the neurological deficit, with complete spinal cord injury most prevalent in the thoracic spine $(70 \%) .^{2-4}$ Bullet trajectory is also closely related to clinical outcomes, with paralysis higher in patients with retained intraspinal bullet fragments. ${ }^{5}$ Patients may also present years later with the development of neurological deficits as the result of bullet migration in the spinal canal.

Ideal management of spinal cord injuries caused by GSWs remains a controversial topic. The most recent literature on the subject advocates conservative management because of the lack of evidence showing that surgical intervention improves outcomes and the high complication rate associated with surgery. However, there remains a subset of injury patterns that have shown improved neurological recovery after surgical decompression and bullet removal as compared with that after conservative management, one of which is cauda equina injury. We present a case of a patient with cauda equina symptoms caused by a migrating retained intraspinal bullet following a GSW. The patient was treated with laminectomy and removal of the bullet. This illustrative case is supplemented by an operative video (Video 1).

VIDEO 1. Clip showing the operation. Click here to view.

\section{Illustrative Case}

\section{History}

An 18-year-old female presented to the emergency department with left flank pain and numbness in her left lower extremity 30 

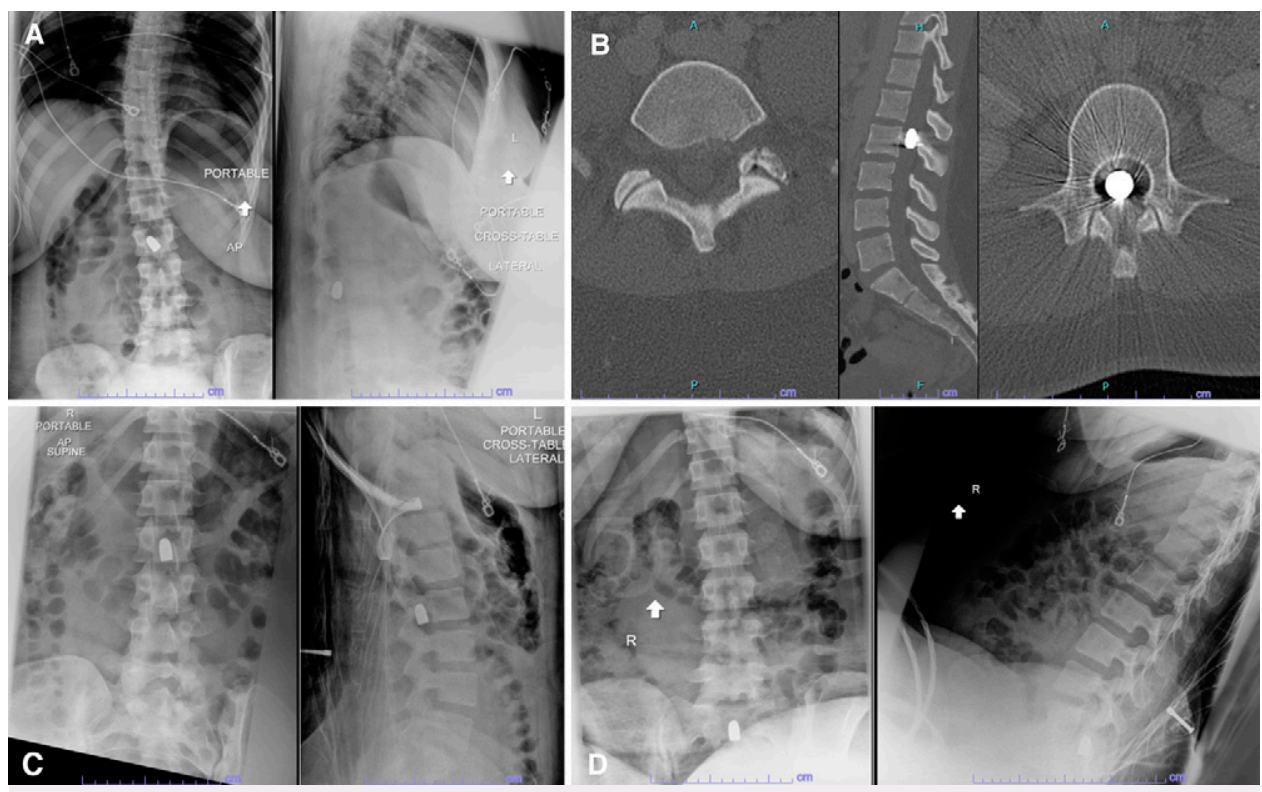

FIG. 1. A: Initial radiographs of the lumbar spine revealing a bullet in the spinal canal at the L2 level.

B: Computed tomography scans of the lumbar spine upon initial presentation demonstrating an S1 left superior articular process fracture extending into the left L5-S1 facet joint and a retained bullet in the spinal canal. C: Radiographs of the lumbar spine 24 hours after presentation demonstrating rotation of the bullet within the spinal canal. D: Radiographs of the lumbar spine 48 hours after presentation demonstrating caudal migration of the bullet to S1. Arrows in the images are radiographic indicators for upright images.

minutes after a GSW to her left lower flank. On arrival, she was hemodynamically stable with an entry wound noted in the distal left flank. Physical examination of the left lower extremity revealed $4 / 5$ strength, except for an ungradable hip flexion secondary to pain. Her right lower extremity demonstrated $3 / 5$ hip flexion and ankle plantarflexion; $1 / 5$ knee flexion, knee extension, and ankle dorsiflexion; and 0/5 great toe extension. Her knee jerk and Achilles reflexes were hyporeflexic bilaterally, and diffuse paresthesia was present to the bilateral lower extremities.

Plain radiographs of the lumbar spine revealed a comminuted fracture of the left iliac crest and a bullet fragment in the spinal canal at the L2 level (Fig. 1A). A computed tomography scan of the lumbar spine demonstrated an $\mathrm{S} 1$ left superior articular process fracture extending into the left L5-S1 facet joint and a fracture of the $\mathrm{L} 5$ vertebral body posterior cortex. A bullet was retained in the spinal canal at the level of $L 2$ (Fig. 1B).

The patient was admitted for serial neurological examinations and placed upright in bed to assess for possible caudal migration of the bullet. Repeat radiographs 24 hours later revealed possible rotation of the bullet at L2 (Fig. 1C), and the decision was made for controlled mobilization of the patient. Repeat radiographs 48 hours from admission revealed caudal migration of the bullet within the spinal canal to S1 (Fig. 1D). The patient subsequently developed urinary retention requiring bladder scans and straight catheterization. She also reported diffuse allodynia to her lower extremities. A decision was made to proceed with operative management because of the neurological exam changes and mobile nature of the bullet in the intradural space.

\section{Operative Course}

The patient was positioned prone on a Wilson frame with the head of the bed elevated to prevent further migration of the bullet proximally. The position of the bullet was confirmed with lateral fluoroscopy (Fig. 2). The surgical site was prepped and draped in routine fashion. A small midline incision was made over S1. Dissection was performed over the $\mathrm{S} 1$ spinous process. The bullet was located

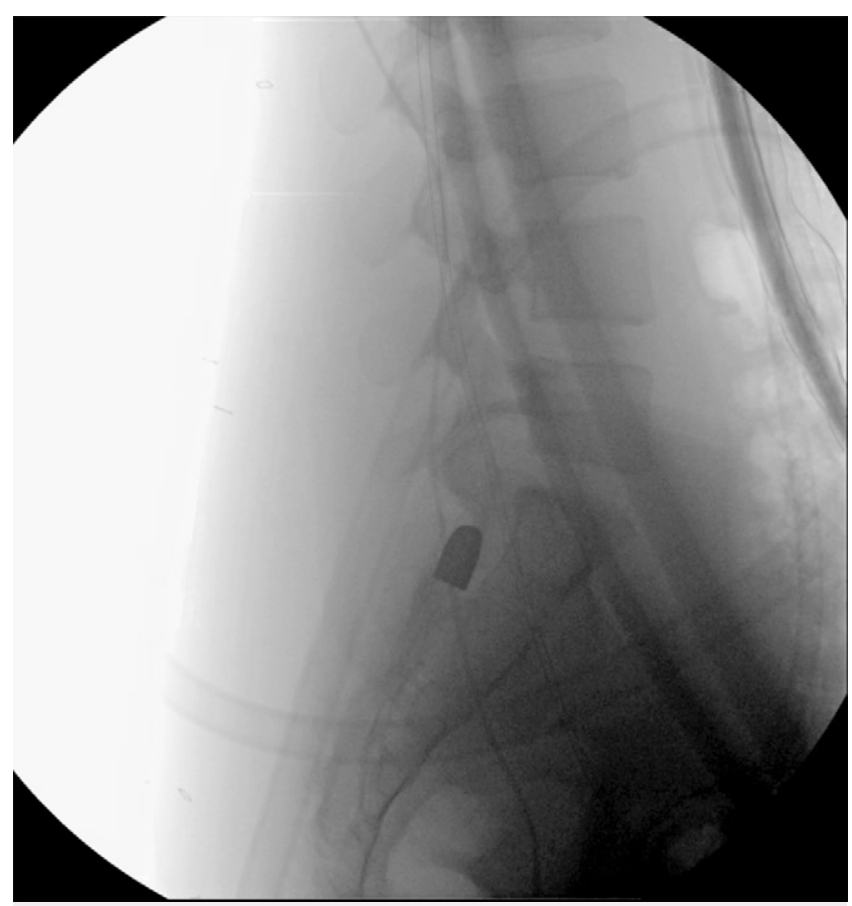

FIG. 2. Intraoperative fluoroscopy confirming anatomical levels. 


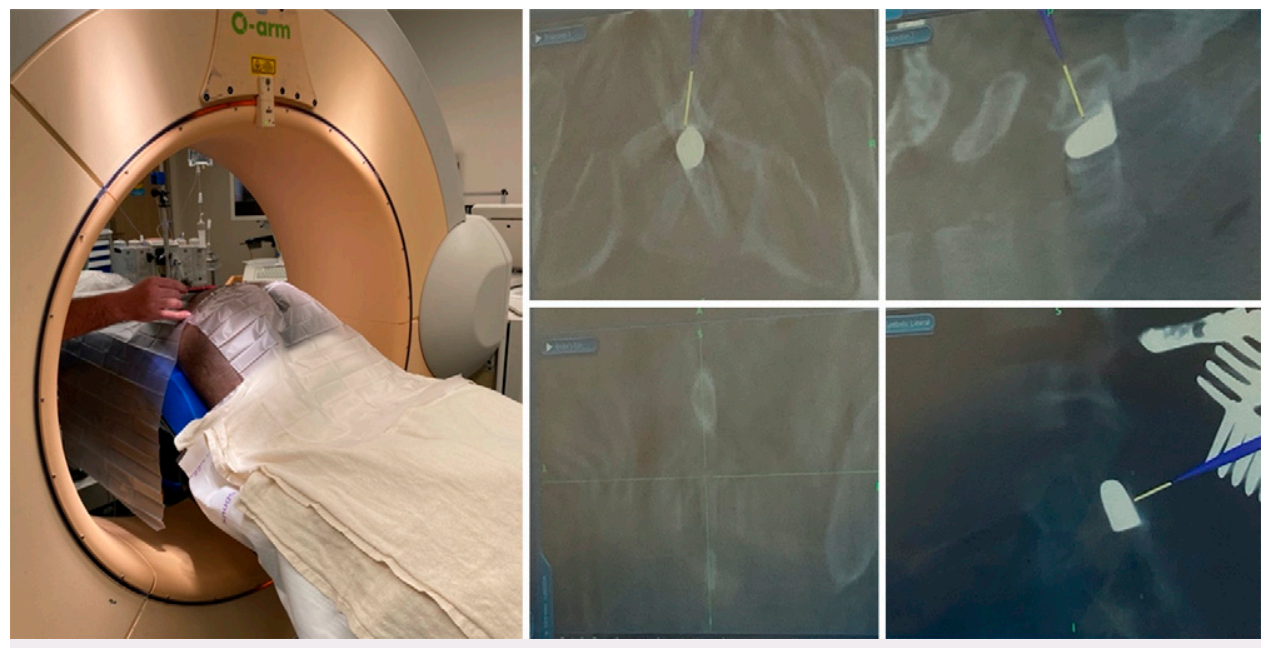

FIG. 3. O-arm navigation was used to identify the exact location of the bullet. Outline of the bullet was marked on S1 lamina using intraoperative navigation.

using navigation, and its location was marked on S1 using a marker (Fig. 3). With the aid of a high-speed burr, a Kerrison rongeur, and a curved curette, the bone over the bullet was removed and the dura was exposed. An operative microscope was then used for the remainder of the case. The dura was tagged with 5-0 sutures (Fig. 4) and then incised. The bullet was visualized, and we attempted to remove it with a micro pituitary rongeur; however, the bullet migrated proximally. Consequently, we excised the ligamentum flavum proximally to the inferior border of L5. At this level, we extended the dural incision, which allowed revisualization of the bullet. We then placed a Penfield 4 dissector (Novo Surgical) proximal to the bullet to prevent further migration of the bullet. Subsequently, we used a micro pituitary rongeur to remove the bullet from the dural sac (Fig. 5A). The bullet was identified as being 9-mm caliber (Fig. 5B). Neuromonitoring throughout the case remained stable. Nurolon 4-0 suture (Ethicon) was used for the dural repair. A Valsalva maneuver was performed without cerebrospinal fluid (CSF) leakage. The dural repair was subsequently reinforced with

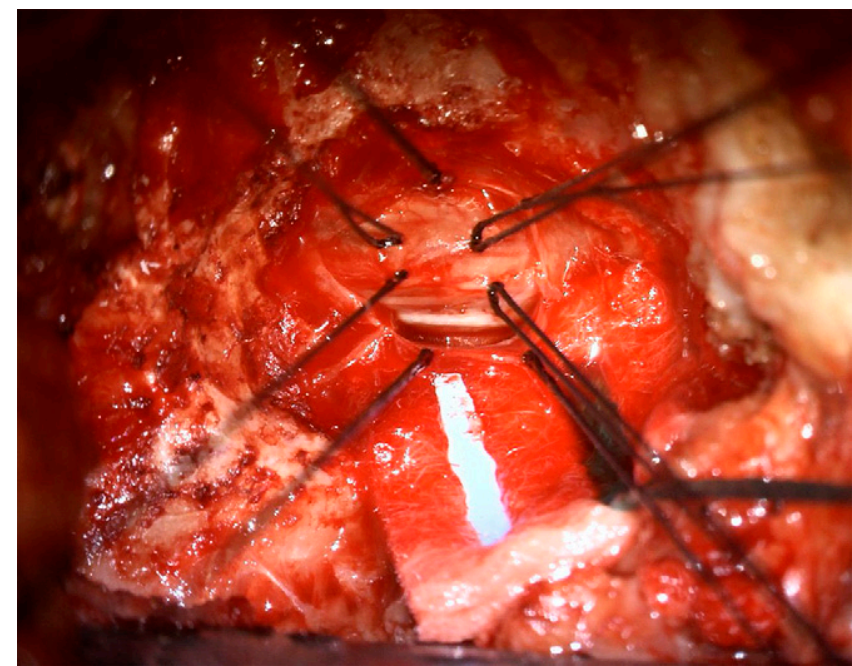

FIG. 4. Intraoperative photograph exhibiting the dural layer tagged with sutures prior to incision.
DuraSeal and DuraGen (Integra LifeSciences). The area was then irrigated and closed in standard fashion (Video 1).

\section{Postoperative Course}

The patient noted immediate improvement of her lower extremity allodynia and paresthesia following surgery. Examination revealed significant improvement, with $5 / 5$ strength to the right lower extremity; $5 / 5$ knee flexion and extension; and 4/5 ankle plantarflexion, dorsiflexion, and great toe extension to the left lower extremity. She was placed on bed rest in a flat position for 48 hours. A Foley catheter that had been placed preoperatively was removed after mobilization; however, the patient remained unable to void spontaneously and required bladder scans with straight catheterizations. She was discharged to a local rehabilitation center on postoperative day 5 with a clinic appointment scheduled for 2 weeks later. At her follow-up appointment, the patient reported resolution of her lower extremity pain and the return of normal bladder function. The results of her motor and sensory examination remained unchanged from discharge.

\section{Discussion}

\section{Observations}

The decision to proceed with surgical decompression and bullet removal in GSWs to the spine continues to be a controversial topic,
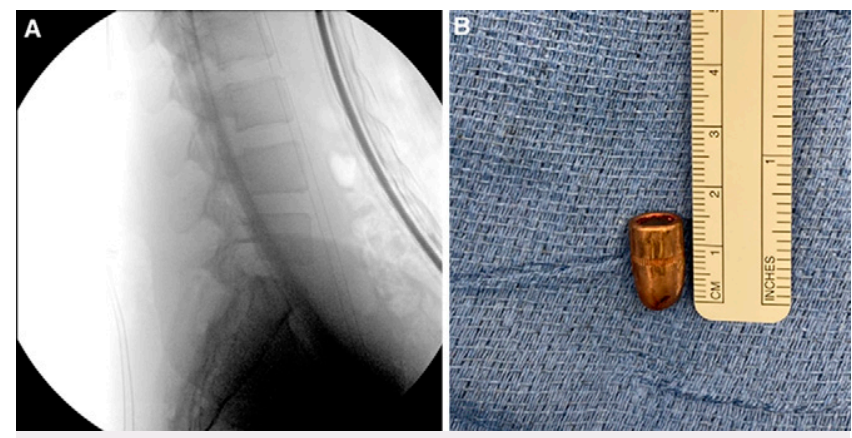

FIG. 5. A: Intraoperative fluoroscopy demonstrating successful removal of the bullet. B: Nine-millimeter caliber bullet after removal from the dural sac. 
with multiple variables that include overall patient status, concomitant injuries, neurological status, spinal stability, and radiographic studies. Two conditions in which the literature supports surgical intervention on the basis of evidence of improved neurological recovery are (1) the presence of progressive neurological deficits and (2) cauda equina lesions. ${ }^{2,3,6-11}$ Neurological improvement in the case of cauda equina is thought to be attributable to the inherent anatomical difference of lumbosacral nerve roots and the potential for peripheral nerve axons to regenerate. ${ }^{2,6}$ Patient symptoms are also a crucial factor in determining whether to proceed with conservative or operative management; thus, thorough serial neurological examinations are of the utmost importance. In the case described, the decision to proceed with bullet removal was based on the symptoms of cauda equina, the development of urinary retention, and migration of the bullet.

A rare complication of retained bullet fragments is the development of neurological symptoms months to years after the injury. Potential causes of this late sequela include bullet migration ${ }^{12}$ and reactive epidural fibrosis induced by the retained fragment. ${ }^{13}$ Intraspinal migration in the lumbosacral spine, although uncommon, is most frequently caudal in nature because of gravity as well as CSF recirculation and respiration mechanics. ${ }^{10}$ Cephalad migration from the lumbosacral spine is limited because of anatomical narrowing of the spinal canal at the level of T10. ${ }^{14}$ However, increased lumbar lordosis may allow cranial migration, which can occur intraoperatively or during patient positioning. ${ }^{10}$ Therefore, preoperative and intraoperative imaging is essential to ensure the correct spinal levels. ${ }^{14,15}$ Conversely, bullet migration can be used to the advantage of the surgeon in preoperative planning. Placing the patient upright or in the reverse Trendelenburg position ${ }^{15}$ can encourage caudal migration of the bullet, permitting relatively safer surgical excision. In the case of our patient, mobilization further enhanced caudal migration of the fragment, likely secondary to movement of spinal motion segments. This allowed surgical removal of the bullet at a relatively safer level (L5/S1) than the initial location of the fragment (L1/L2/conus medullaris). If the bullet is in close proximity to the dura or intrathecal or if there is high potential for migration, surgical intervention is preferred. ${ }^{4,9,16}$

Plumbism from retained lead-containing missiles is another unusual occurrence that has rarely been reported in the literature. Lead fragments in direct contact with synovial fluid, a pseudocyst, or the disc space are more often associated with lead toxicity. However, the overall number of reported cases remains small, and the lack of evidence in the literature does not support retained lead fragments as the sole indication for surgical removal in the acute setting. ${ }^{7}$ Lead toxicity, when it does occur, is typically a chronic and insidious process. Patients with retained bullet fragments should be followed closely for the development of lead toxicity symptoms because of their increased risk of plumbism. ${ }^{17}$ Patients diagnosed with lead intoxication require chelation therapy in conjunction with surgical management for successful resolution of symptoms. ${ }^{18}$

\section{Lessons}

Management of spinal cord injuries caused by GSWs continues to be a controversial topic. Thorough neurological examinations and serial imaging are imperative in the treatment decision-making process. In the case of cauda equina injuries or the presence of progressive neurological deficits, treatment with surgical decompression and bullet removal has been shown to improve symptoms. Migration of the bullet within the spinal canal can result in progressive neurological deficits in the future; however, bullet migration can also be used to the surgeon's advantage, allowing removal at a safer anatomical level. If the decision is made to remove the bullet, special attention should be paid to bullet migration while positioning the patient in the operating room and throughout the procedure.

\section{References}

1. National Spinal Cord Injury Statistical Center, University of Alabama at Birmingham. 2019 Annual Statistical Report-Complete Public Version. National Spinal Cord Injury Statistical Center, University of Alabama at Birmingham.

2. Kitchel SH. Current treatment of gunshot wounds to the spine. Clin Orthop Relat Res. 2003;(408):115-119.

3. Waters RL, Sie IH. Spinal cord injuries from gunshot wounds to the spine. Clin Orthop Relat Res. 2003;(408):120-125.

4. de Barros Filho TE, Cristante AF, Marcon RM, et al. Gunshot injuries in the spine. Spinal Cord. 2014;52(7):504-510.

5. Chittiboina $P$, Banerjee $A D$, Zhang $S$, et al. How bullet trajectory affects outcomes of civilian gunshot injury to the spine. J Clin Neurosci. 2011;18(12):1630-1633.

6. Sidhu GS, Ghag A, Prokuski V, et al. Civilian gunshot injuries of the spinal cord: a systematic review of the current literature. Clin Orthop Relat Res. 2013;471(12):3945-3955.

7. Isiklar ZU, Lindsey RW. Gunshot wounds to the spine. Injury. 1998;29(suppl 1):SA7-SA12.

8. Bono CM, Heary RF. Gunshot wounds to the spine. Spine J. 2004;4(2):230-240.

9. Waters RL, Adkins RH. The effects of removal of bullet fragments retained in the spinal canal. A collaborative study by the National Spinal Cord Injury Model Systems. Spine (Phila Pa 1976). 1991;16(8):934-939.

10. Baldawa S, Shivpuje V. Migratory low velocity intradural lumbosacral spinal bullet causing cauda equina syndrome: report of a case and review of literature. Eur Spine J. 2017;26(suppl 1): 128-135.

11. Bumpass DB, Buchowski JM, Park A, et al. An update on civilian spinal gunshot wounds: treatment, neurological recovery, and complications. Spine (Phila Pa 1976). 2015;40(7): 450-461.

12. Siddiqui MI, Hawksworth SA, Sun DY. Removal of migrating lumbar spine bullet: case report and surgical video. World Neurosurg. 2019;131:62-64.

13. Ajmal S, Enam SA, Shamim MS. Neurogenic claudication and radiculopathy as delayed presentations of retained spinal bullet. Spine J. 2009;9(10):e5-e8.

14. Koban O, Çal H, Ekşi MŞ, et al. Migrating bullet in the thecal sac at the level of the conus medullaris without neurological deficit. J Clin Neurosci. 2016;29:185-188.

15. Ben-Galim P, Reitman CA. Intrathecal migratory foreign body without neurological deficit after a gunshot wound. Spine $J$. 2008;8(2):404-407.

16. Kuijlen JM, Herpers MJ, Beuls EA. Neurogenic claudication, a delayed complication of a retained bullet. Spine (Phila Pa 1976). 1997;22(8):910-914.

17. Linden MA, Manton WI, Stewart RM, et al. Lead poisoning from retained bullets. Pathogenesis, diagnosis, and management. Ann Surg. 1982;195(3):305-313.

18. Towner JE, Pieters TA, Maurer PK. Lead toxicity from intradiscal retained bullet fragment: management considerations and recommendations. World Neurosurg. 2020;141:377-382.

\section{Disclosures}

The authors report no conflict of interest concerning the materials or methods used in this study or the findings specified in this paper. 


\section{Author Contributions}

Conception and design: Salari, de los Cobos, Powers, Behrens.

Acquisition of data: Salari, Behrens. Analysis and interpretation of data: Behrens. Drafting the article: de los Cobos, Powers, Behrens. Critically revising the article: all authors. Reviewed submitted version of manuscript: all authors. Approved the final version of the manuscript on behalf of all authors: Salari. Statistical analysis: Behrens.

Administrative/technical/material support: Salari, de los Cobos, Powers, Behrens. Study supervision: Salari.

\section{Supplemental Information}

Videos

Video 1. https://vimeo.com/523802897.

\section{Correspondence}

Pooria Salari: Saint Louis University School of Medicine, St. Louis, MO. pooria.salari@health.slu.edu. 